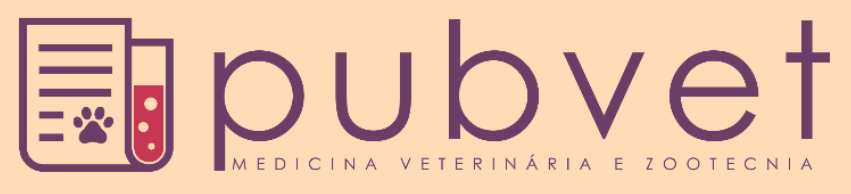

https://doi.org/10.31533/pubvet.v14n5a580.1-11

\title{
Efeito da embalagem em papel filme ou a vácuo sobre características antes e após a maturação da carne de bovinos terminados em confinamento
}

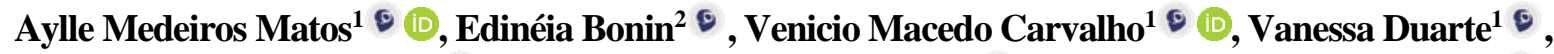

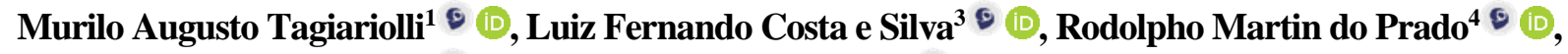 \\ Ana Carolina Pelaes Vital ${ }^{\circ} \mathbb{D}$, Ivanor Nunes do Prado ${ }^{\circ} \circ$ (D) $^{\circ}$ \\ ${ }^{I}$ Alunos do Programa de Pós Graduação em Zootecnia da Universidade Estadual de Maringá - CEP - 87.020-900, Maringá, Paraná, Brasil. \\ ${ }^{2}$ Alunos do Programa de Pós Graduação em Ciências de Alimentos da Universidade Estadual de Maringá-CEP-87.020-900, Maringá, Paraná, Brasil. \\ ${ }^{3}$ Pesquisador da Alltech - Edifício Átrium - Empresarial - Torra Norte, Av. Advogado Horácio Raccanello Filho, 4660 - Sala 1705 - Zona 10, \\ Maringá, Paraná, Brasil, CEP - 87030-405 \\ ${ }^{4}$ Professor do Programa de Pós Graduação em Zootecnia e Ciências de Alimentos da Universidade Estadual de Maringá - Maringá, Paraná, Brasil. \\ Autor para correspondência: Ivanor Nunes do Prado. E-mail: inprado@uem.br
}

Resumo. Este trabalho foi realizado para avaliar a influência do método de embalagem (em bandeja envolta em papel filme ou a vácuo) sobre o $\mathrm{pH}$, coloração, textura, oxidação lipídica e atividade antioxidante da carne bovina antes da maturação (dia 1) e maturada durante 3, 7 e 14 dias. As amostras de carne foram obtidas de 36 animais terminados em confinamento durante 84 dias. Após a coleta, Longissimus dorsi foi filetado em amostras de $2,5 \mathrm{~cm}$ e embaladas em bandeja envoltas em papel filme ou a vácuo durante 3, 7 e 14 dias. Carnes embaladas a vácuo apresentaram $\mathrm{pH}$ mais baixo $(\mathrm{P}<0.001)$. $\mathrm{O}$ tempo de maturação reduziu $(\mathrm{P}<0,001) \mathrm{pH}$ da carne em ambas as embalagens. As perdas por cocção foram maiores $(\mathrm{P}<0,001)$ para as carnes embaladas a vácuo e foram reduzidas com $\mathrm{o}$ tempo de maturação. Os valores de $\mathrm{L}^{*}$ não foram alterados $(\mathrm{P}>0,05)$ pelas embalagens, mas foram reduzidos pela maturação. Os valores de $a^{*}$ não foram alterados $(P>0,05)$ pelas embalagens, mas foram aumentados pela maturação. A textura e oxidação lipídica não foram alteradas $(\mathrm{P}>0,05)$ pelas embalagens, mas a textura melhorou com a maturação, enquanto que a oxidação lipídica aumentou. Os métodos de embalagens e os dias de maturação não tiveram efeito $(P>0,05)$ na atividade antioxidante da carne medida por três diferentes metodologias (ABTS, DPPH e FRAP).

Palavras chave: Antioxidantes, carne bovina, embalagem, Longissimus, oxidação de lipídeos

\section{Effect of packaging on film paper or in vacuum on characteristics before and after aging of meat from bulls finished in feedlot}

Abstract. This work was conducted to evaluate the influence of the packaging method (in
a tray wrapped in film paper or vacuum) on $\mathrm{pH}$, color, texture, lipid oxidation, and
antioxidant activity of beef before aging (day 1$)$ and aging for 3,7 and 14 days. The samples
of meat were obtained from 36 animals finished in feedlot for 84 days. After collection,
Longissimus dorsi was filleted in $2.5 \mathrm{~cm}$ samples packed in a tray wrapped in film or
vacuum for 3,7 and 14 days. Meats in vacuum packaging showed lower $\mathrm{pH}(\mathrm{P}<0.001)$.
The aging time reduced $(\mathrm{P}<0.001) \mathrm{pH}$ value of the meat in both methodologies. Cooking
losses were higher $(\mathrm{P}<0.001)$ for vacuum-packed meats and were reduced with aging time.
The $\mathrm{L}^{*}$ values were not changed $(\mathrm{P}>0.05)$ by the packaging, but were reduced by aging.
The values of a* were not altered $(\mathrm{P}>0.05)$ by the packaging, but were increased by aging.
The texture and lipid oxidation were not altered $(\mathrm{P}>0.05)$ by the packaging, but the texture
was improved by aging, while the lipid oxidation increased. The packaging and aging days 
had no effect $(\mathrm{P}>0.05)$ on antioxidant activity of the meat as measured by three methodologies (ABTS, DPPH and FRAP).

keyword: Antioxidants, meat cattle, packaging, Longissimus, lipids oxidation

\title{
Efecto del envase en película de filme o al vacío en características antes y después de la maduración de la carne del ganado engordado en cebadero
}

\begin{abstract}
Resumen. Este trabajo se llevó a cabo para evaluar la influencia del método de envasado (en bandeja envuelta en película de filme o al vacío) sobre el $\mathrm{pH}$, el color, la textura, la oxidación de lípidos y la actividad antioxidante de la carne antes de la maduración (día 1) y madurada durante 3, 7 y 14 días. La carne se obtuvo de 36 animales terminados en confinamiento durante 84 días. Después de la recolección, se fileteó el Longissimus dorsi en muestras de 2,5 cm y envasadas en una bandeja envuelta en película o al vacío durante 3, 7 y 14 días. Las carnes envasadas al vacío mostraron un pH más bajo ( $\mathrm{P}<0.001)$. El tiempo de maduración redujo $(\mathrm{P}<0.001)$ pH de la carne en ambos tipos de envasado. Las pérdidas de cocción fueron mayores $(\mathrm{P}<0,001)$ para las carnes envasadas al vacío y se redujeron con el tiempo de maduración. Los valores de $\mathrm{L}^{*}$ no fueron modificados $(\mathrm{P}>0,05)$ por el envase, pero fueron reducidos por la maduración. Los valores de a* no fueron alterados $(\mathrm{P}>$ $0,05)$ por el envase, pero aumentaron $(\mathrm{P}<0,001)$ por la maduración. El envase no alteró $(\mathrm{P}>0,05)$ la textura y la oxidación de los lípidos, pero la textura mejoró con la maduración, mientras que la oxidación de los lípidos aumentó. Los días de envasado y maduración no tuvieron efecto $(\mathrm{P}>0,05)$ sobre la actividad antioxidante de la carne medida por tres metodologías (ABTS, DPPH y FRAP).
\end{abstract}

Palabras clave: Antioxidantes, carne bovina, embalaje, Longissimus, oxidación de lípidos

\section{Introdução}

Para satisfazer as demandas dos consumidores em relação à comercialização de carne garantindo a segurança e alta qualidade, a indústria de alimentos precisa estar preocupada com mínimo processamento do produto pronto para consumo ou exigindo pouco preparo destes produtos.

A carne bovina é comercializada de várias maneiras e em vários pontos de venda. De modo geral, o comprador, mais precisamente, a compradora de carne adquire a mesma preferencialmente em supermercados ou açougues (Guerrero et al., 2018; Vital et al., 2018). No entanto, a compra da carne é realizada preferencialmente como corte a fresco pelo atendente ou em bandejas (Eiras et al., 2017). O comportamento dos consumidores está adquirindo outro perfil com a industrialização das economias em todo o mundo. Desta forma, novas formas de apresentação da carne são necessárias para atrair a atenção destes consumidores (Realini \& Marcos, 2014).

Os métodos de embalagens envolvem novos desafios para a sociedade moderna e esta exige uma abordagem diferenciada, inclui a mobilidade dos bens de consumo, o mercado mundial, o tempo de prateleira dos produtos in natura ou industrializados, a conveniência para comercialização das mercadorias, a segurança sanitária dos produtos, cuidado ao meio ambiente, as marcas registradas e consolidadas e o descarte dos resíduos, entre outros (Hocquette, 2015; Kerry, 2014). A otimização das embalagens (formato, tamanho, cores, facilidade de leitura das etiquetas e métodos de embalagens) podem auxiliar o consumidor no momento da compra, assim novas embalagens estão sendo desenvolvidas e disseminadas para manter a qualidade do produto e evitar desperdícios dos alimentos (Realini \& Marcos, 2014). Além disso, a segurança alimentar está sendo prioritária para os atuais consumidores. Ainda, a demanda atual dos consumidores está sendo por produtos com menor processamento, mais naturais, frescos, aspectos recentes e encontrados facilmente para aquisição. Estes aspectos de demanda revela uma oportunidade ímpar para as indústrias de embalagens apresentarem soluções inovadoras aos diferentes segmentos de comercialização para satisfação e bem estar dos consumidores. A embalagem deve ter, também, baixo custo para não inviabilizar o valor de comercialização da carne.

As embalagens chamadas de ativas ou inteligentes são bons exemplos dos sistemas de inovações que está ocorrendo com a globalização da economia (Realini \& Marcos, 2014). Todavia, as embalagens tradicionais ainda são usadas em larga escala como, por exemplo, a comercialização de carnes em bandejas de poliestireno, envoltas em papel filme (mais barata) e as embalagens a vácuo (maior tempo de prateleira). 
Portanto, este trabalho foi realizado para avaliar os efeitos do método de embalar a carne (bandeja de poliestireno envolta em papel filme) ou a vácuo, antes e após a maturação, da carne de bovinos jovens terminados em confinamento e abatidos aos 24 meses de idade.

\section{Material e métodos}

\section{Comitê de ética, local e animais}

Este experimento foi aprovado pelo Departamento de Zootecnia e pelo Comitê de Ética em Pesquisa Animal da Universidade Estadual de Maringá, e seguiu os princípios da pesquisa de biomédica animal sob o número 081/2014.

O confinamento dos bovinos foi realizado no setor de Bovinocultura de Corte (Rosa \& Pedro) na Fazenda Experimental de Iguatemi da Universidade Estadual de Maringá, situada em Maringá, estado do Paraná, Sul do Brasil. As análises das características da carne foram realizadas no Laboratório de Qualidade da Carne da Universidade Estadual de Maringá.

Um total de 36 bovinos mestiços (Europeus $v$ s. Nelore), não castrados com $24 \pm 3.2$ meses de idade e com peso vivo médio inicial de $385,5 \pm 3,84 \mathrm{~kg}$ foram usados em um arranjo completamente casualizado.

\section{Abate dos animais}

Ao final do período experimental (84 dias), os bovinos foram pesados com 16 horas de jejum (média de $539,3 \pm 6,32 \mathrm{~kg}$ ) e transportados para um abatedouro comercial (cidade de Campo Mourão, estado do Paraná, Brasil). Os bovinos foram abatidos seguindo as práticas usuais de abate usadas pela indústria brasileira. $\mathrm{Na}$ sequência, as carcaças foram divididas ao meio a partir do esterno até a espinha, em duas partes semelhantes. Depois disso, as meias carcaças foram lavadas, identificadas e estocadas em câmaras frias a $+4^{\circ} \mathrm{C}$, onde as mesmas permaneceram durante 24 horas.

\section{Amostragem da carne}

No dia seguinte após o abate, o Longissimus dorsi entre a $7^{\mathrm{a}}$ e $12^{\mathrm{a}}$ costelas foi removido e transportado para o Laboratório de Qualidade de Carne da Universidade Estadual de Maringá para o processamento a realização das análises.

\section{Tratamentos}

As carnes foram embaladas em dois métodos: bandeja de poliestireno envoltas em papel filme e a vácuo antes da maturação e nos dias 3, 7 e 14 de maturação.

\section{Maturação da carne}

As amostras da carne do primeiro dia foram analisadas imediatamente. As demais amostras foram maturadas durante três, sete e 14 dias embaladas em bandejas envoltas em papel filme ou a vácuo. Para a embalagem em papel filme foi usado o sistema convencional de venda da carne nos supermercados, isto é, carne embalada em bandeja de poliestireno envolta em papel filme e colocada em display. As carnes embaladas a vácuo foram embaladas em embalagens próprias para este sistema $(25 \times 14 \times 0,8 \mathrm{~cm})$, transparentes (poliamida/polietileno) de $120 \mu \mathrm{m}$, com $1 \mathrm{~cm}^{3} / \mathrm{m}^{2}$ por $24 \mathrm{~h}$, permeabilidade de $\mathrm{CO}_{2}$ e $3 \mathrm{~cm}^{3} / \mathrm{m}^{2}$ por $24 \mathrm{~h}$ permeabilidade de $\mathrm{CO}_{2}$ a $4^{\circ} \mathrm{C}$ e $75 \%$ de umidade relativa, com $3 \mathrm{~g} / \mathrm{m}^{2}$ por $24 \mathrm{~h}$ com taxa de transmissão de vapor de água a $38^{\circ} \mathrm{C}$ e $100 \%$ de umidade relativa; $97^{\circ} \mathrm{C}$ Vicat de temperatura e $1,3 \mathrm{~g}$ força de resistência e selada usando um equipamento Sulpack SVC 620 (VAC). As amostras foram colocadas em display $\left(4+1^{\circ} \mathrm{C}\right)$ durante três, sete e 14 dias, simulando a típica comercialização de carne no Brasil com luz artificial de 50/50 lâmpada siliconada de diodo (LED), 4,8 W, durante $12 \mathrm{~h} / \mathrm{dia}$.

$p H$

O pH foi mensurado no Longissimus thoracis (LT) usando um pHmetro (Hanna - HI99163, Romania Europe) com o eletrodo de penetração colocado na $12^{\text {th }}$ vértebra lombar. O pHmetro foi calibrado a $20^{\circ} \mathrm{C}$ usando um padrão tampão $\mathrm{pH} 4,0$ e 7,0.

\section{Coloração}

A coloração da carne foi avaliada após 30 minutos de exposição ao ar no primeiro dia e aos 3, 7 e 14 dias de display, usando sistema CIEL*a*b* com um colorímetro Minolta CR-400 (Japan) (com um ângulo de visão de $10^{\circ}$, iluminate D65 e abertura de oito $\mathrm{mm}$ com cone fechado). Seis medidas foram tomadas ao acaso e registradas por bife obtendo, assim, a luminosidade $\left(\mathrm{L}^{*}\right)$, tom de vermelho $\left(\mathrm{a}^{*}\right)$ e tom de amarelo $\left(\mathrm{b}^{*}\right)$. 
Warner-Bratzler Shear Force - WBSF - Textura

A textura dos bifes previamente cozidos foi analisada usando um texturômetro TA.XT Plus (Texture Technologies 15 Corp., UK) equipado com uma lâmina da Warner-Bratzler, velocidade de $1 \mathrm{~mm}$ e carga de $5 \mathrm{~kg}$, seguindo protocolo descrito por Honikel (1998). Os bifes foram cortados em pedaços retangulares de $2,5 \mathrm{~cm}^{2}$ (seis bifes por animal) perpendicularmente à direção das fibras musculares.

\section{Oxidação lipídica - Substância Reativa ao Ácido Tiobarbitúrico - TBARS}

A oxidação de lipídeos foi medida pela concentração de malonaldeído (MDA) na carne. A oxidação foi quantificada usando o ensaio com substância reativa do ácido tiobarbitúrico (TBARS) de acordo com Vital et al. (2016). As amostras da carne (5 gramas) foram misturadas com a solução de TCA (7,5\%, 0,1\% EDTA e $0,1 \%$ de ácido gálico) $(10 \mathrm{ml})$, homogeneizada usando um Ultra-Turrax, e centrifugada a $4^{\circ} \mathrm{C}$ durante 15 minutos a $4.000 \mathrm{rpm}$. O sobrenadante foi filtrado e misturado ao reagente de TBARS (1:1 v/v). A solução foi aquecida em banho Maria $\left(100^{\circ} \mathrm{C}\right)$ durante 15 minutos, resfriada e lida em espectrofotômetro com um comprimento de onda de $540 \mathrm{~nm}$ em comparação com uma curva padrão. Os resultados foram expressos como mg de MDA $/ \mathrm{kg}^{-1}$ de carne crua. A oxidação de lipídeos foi avaliada antes da maturação e aos três, sete e 14 dias de maturação.

\section{Atividade antioxidante}

\section{Extratos bioativos da carne}

Os extratos bioativos da carne $(1: 1 \mathrm{w} / \mathrm{v}$ com metanol) foram obtidos de acordo com a metodologia descrita por Vital et al. (2016) usando um Ultra-Turrax (IKA ${ }^{\circledR}$ - T10, USA), seguido pela centrifugação (4.000 rpm, $15 \mathrm{~min}$ ) e filtragem (papel filtro com gramatura $-80 \mathrm{~g} / \mathrm{m}^{2}$, espessura $-205 \mu \mathrm{m}$, poros $-14 \mu \mathrm{m}$ ). A capacidade antioxidante da carne foi avaliada usando o 2,2-diphenyl-1-picrylhydrazyl (DPPH) e 2,2'-azino-bis (3ethylbenzthiazoline-6-sulfonico ácido) (ABTS).

\section{Atividade Antioxidante pelo Método de 2,2-difenil-1-picrihidrazil-DPPH}

A atividade de sequestro do radical livre com DPPH foi medida de acordo com a metodologia descrita por Li et al. (2009), com modificações realizadas por Vital et al. (2016). Os extratos da carne (150 $\mu \mathrm{L})$ foram homogeneizados com uma solução metanólica de DPPH $(60 \mu \mathrm{M})$ e deixados no escuro durante 30 min para que ocorresse a reação. A absorbância foi medida a $515 \mathrm{~nm}$ de comprimento de onda. A atividade antioxidante foi calculada como:

DPPH $(\%)=(1-($ Amostra $\mathrm{t}=0 /$ Amostra $\mathrm{t})) * 100$

Onde: Amostra $\mathrm{t}=0$ é a absorbância da amostra no tempo zero, e Amostra té a absorbância da amostra aos 30 minutos.

\section{Atividade Antioxidante pelo Método 2,2-azino-bis-etilbenziazolina-6-ácido Sulfônico-ABTS}

O ensaio de ABTS foi conduzido de acordo com a metodologia de Re et al. (1999) com modificações de Vital et al. (2016). O ABTS ${ }^{+}$foi gerado pela interação de $7 \mathrm{mM}$ ABTS $(5 \mathrm{~mL})$ com $140 \mathrm{mM}$ de persufalto de potássio $(88 \mu \mathrm{L})$. A mistura foi incubada no escuro a $25^{\circ} \mathrm{C}$ durante $16 \mathrm{~h}$. O radical ativado do ABTS foi diluído com etanol para uma absorbância $0,70 \pm 0,02$ a $734 \mathrm{~nm}$. A atividade de sequestro de radical foi mensurada a $734 \mathrm{~nm}$. O extrato da carne $(40 \mu \mathrm{L})$ foi misturado com a solução $\operatorname{ABTS}^{+}(1960 \mu \mathrm{L})$ e as leituras foram feita após 6 min no escuro. A atividade de sequestro de radical foi calculada como:

$\operatorname{ABTS}(\%)=(1-($ Amostra $\mathrm{t}=0 /$ Amostra $\mathrm{t})) * 100$

Onde: Amostra $\mathrm{t}=0$ é a absorbância da amostra no tempo zero, e Amostra té a absorbância da amostra aos seis minutos.

Atividade Antioxidante pelo Método de Redução do Complexo Férrico-Tripiridiltriazine para a Forma Ferrosa-FRAP

A metodologia de FRAP foi usada de acordo com as recomendações de Zhu et al. (2002). O extrato da carne $(250 \mu \mathrm{L})$ foi misturado com $50 \mathrm{mM}$ de tampão de fosfato de sódio $\mathrm{pH} 7(1,25 \mathrm{~mL})$ e $1 \%$ de ferricianida de potássio $(1,25 \mathrm{~mL})$ e incubado a $50^{\circ} \mathrm{C}$ durante 20 minutos. Em seguida, $10 \%$ de ácido tricloroacético $(1,25 \mathrm{~mL})$ foram adicionados e a mistura foi centrifugada a $4.000 \mathrm{rpm}$ durante 10 minutos. A camada superior $(2,5 \mathrm{~mL})$ foi misturada com cloreto de ferro $(500 \mu \mathrm{L})$ e a absorbância foi medida a $700 \mathrm{~nm}$. Os resultados foram expressos como equivalente de óleo de ácido gálico (GAE) $/ \mathrm{g}^{-1}$ de carne. $\mathrm{O}$ ácido gálico $\left(0-300 \mathrm{mg} / \mathrm{L}^{-}\right.$ $\left.{ }^{1}\right)$ foi usado para estabelecer a curva padrão. 


\section{Resultados e discussão}

$p H$

As carnes foram embaladas em bandejas de poliestireno (envoltas em papel filme) ou a vácuo. As carnes embaladas a vácuo, com exceção do primeiro dia tiveram menor $\mathrm{pH}$ (Tabela 1). $\mathrm{O}$ valor do $\mathrm{pH}$ da carne, independentemente, do tipo de embalagem variou de 5,77 a 6,15. A embalagem a vácuo auxiliou para proteger a carne na redução do $\mathrm{pH}$, mantendo os valores estáveis durante o decorrer dos dias de análise. A embalagem a vácuo reduz o contato da carne com o oxigênio, assim, reduzindo a oxidação lipídica e proteica (Realini \& Marcos, 2014).

Por outro lado, o tempo de maturação reduziu $(\mathrm{P}<0,001)$ o $\mathrm{pH}$ da carne para ambas as embalagens, entre o primeiro e sétimo dia, passando de 6,0 para 5,7. No entanto, no décimo quarto dia de maturação, o $\mathrm{pH}$ da carne embalada em papel filme foi elevado $(6,2)$. Desta forma, podemos deduzir que o tempo de maturação até sete dias reduz o pH carne. Para maior tempo de maturação (14 dias), o pH foi estabilizado na carne embalada a vácuo, mas aumentou para a carne embalada em papel filme. Assim, o tempo de maturação até sete dias seria ideal para o valor do $\mathrm{pH}$, mas aos 14 dias de maturação, o efeito é nocivo.

Tabela 1. Efeito do método de embalagem e tempo de maturação sobre o pH da carne de bovinos terminados em confinamento

\begin{tabular}{lccccc}
\hline \multirow{2}{*}{ Dias } & \multicolumn{2}{c}{ Métodos de embalagens } & & \multirow{2}{*}{ P $>$ Valor } \\
\cline { 2 - 3 } & Filme & Vácuo & & 0,992 \\
3 & $5,99 \mathrm{~A}$ & $5,99^{\mathrm{a}}$ & 0,040 & 0,001 \\
7 & $5,82 \mathrm{~B}$ & $5,72 \mathrm{~B}$ & 0,028 & 0,001 \\
14 & $5,70 \mathrm{~B}$ & $5,77 \mathrm{~B}$ & 0,026 & 0,001 \\
\hline EPM & $6,15 \mathrm{~A}$ & $5,70 \mathrm{~B}$ & 0,060 & \\
$\mathrm{P}<$ Valor & 0,035 & 0,042 & & \\
\hline
\end{tabular}

*Erro padrão da média. Médias seguidas de letras maiúsculas nas colunas são deferentes $(\mathrm{P}<0,05)$.

O valor médio do $\mathrm{pH}$ da carne $(6,0)$, embora aceitável, pode ser considerado alto. De modo geral, o $\mathrm{pH}$ da carne bovina oscila entre 5,5 a 5,7 (Ornaghi et al., 2020; Page et al., 2001). O valor de pH relativamente alto observado, neste experimento, pode ser explicado, em parte, pelo grupo genético usado ( $1 \frac{1}{2}$ Europeu $v s$. $1 / 2$ Nelore). De modo geral, animais com sangue zebuíno apresentam um valor de $\mathrm{pH}$ em razão do comportamento agressivo destes animais no transporte e no momento do abate (Maggioni et al., 2012). Françozo et al. (2013) e Rotta et al. (2014) observaram pH acima de 6,2 para bovinos mestiços Nelores terminados em confinamento e abatidos de forma semelhante ao deste experimento.

\section{Perdas por cocção}

Antes da maturação as perdas por cocção da carne foram semelhantes para as ambas embalagens (Tabela 2). No entanto, ao longo do período de maturação, a carne embalada a vácuo apresentou maior $(\mathrm{P}<0,001)$ perda de água quando comparada com a carne embalada em papel filme. Ao longo do período de maturação a carne embalada em papel filme fica com maior exposição ao meio ambiente, enquanto que a carne embalada a vácuo é mantida hermeticamente protegida. Desta forma, todo exsudato da carne embalada a vácuo é mantido dentro da embalagem, enquanto que para a carne embalada em papel filme pode ocorrer evaporação da água durante o armazenamento. Assim, essa diferença entre os dois métodos de embalagens pode ser explicado pela evaporação da água que ocorre durante o display em vitrine, simulando o sistema de venda de carne em bandeja envolta em papel filme.

As perdas por cocção para a carne embalada em papel filme reduziram-se $(\mathrm{P}<0,001)$ nos primeiros três dias de maturação (Tabela 2). Na sequência, as perdas manterem-se estabilizadas, próximas de $22 \%$. Para a carne embalada a vácuo, as perdas foram menores $(\mathrm{P}<0,001)$ no terceiro e sétimo dia de maturação, intermediárias antes da maturação e maiores no décimo quarto dia de maturação. A redução nas perdas de água nos dois primeiros dias de maturação (três e sete) pode estar relacionada às elevadas perdas antes da maturação $(31,3 \%)$. De modo geral, as perdas de água por cocção variam de 20 a $25 \%$ na carne bovina durante o tempo de maturação (Rivaroli et al., 2016; Rivaroli et al., 2020). Ornaghi et al. (2020) também observaram uma elevada perda de água por cocção (acima de 33\%) para a carne de bovinos terminados em confinamento e alimentados com dietas semelhantes à deste estudo. 
Tabela 2. Efeito do método de embalagem e tempo de maturação sobre as perdas por cocção da carne de bovinos terminados em confinamento

\begin{tabular}{lccccc}
\hline \multirow{2}{*}{ Dias } & \multicolumn{2}{c}{ Métodos de embalagens } & & PPM* & P Valor \\
\cline { 2 - 3 } & Filme & Vácuo & & 0,492 & 0,982 \\
3 & $31,34 \mathrm{~A}$ & $31,34 \mathrm{~A}$ & & 0,001 \\
7 & $22,28 \mathrm{~B}$ & $27,02 \mathrm{~B}$ & 0,173 & 0,001 \\
14 & $23,86 \mathrm{~B}$ & $26,83 \mathrm{~B}$ & 0,142 & 0,001 \\
\hline EPM & $21,90 \mathrm{~B}$ & $38,54 \mathrm{C}$ & 0,150 & \\
P V Valor & 0,152 & 0,144 & & \\
\hline
\end{tabular}

*Erro padrão da média. Médias seguidas de letras maiúsculas nas colunas são deferentes $(\mathrm{P}<0,05)$.

\section{Medida instrumental da coloração da carne}

O parâmetro L* (luminosidade) mede a variação da coloração da carne entre clara e escura. Valores maiores significam carnes mais claras e valores menores significam carnes mais escuras. $\mathrm{O}$ valor ideal de $\mathrm{L}^{*}$ na carne bovina está compreendido entre 38 a 40 pontos (Faustman et al., 2010; Mancini \& Hunt, 2005).

Antes da maturação (dia 1) e três dias mais tarde, o método de embalagem não teve efeito $(P>0,05)$ no valor de $L^{*}$ (Tabela 3). Todavia, os valores de $\mathrm{L}^{*}$ observados no sétimo e décimo quarto dia de maturação foram superiores $(\mathrm{P}<0,001)$ para as carnes embaladas em papel filme. Desta forma, a embalagem em papel filme não protegeu a carne da oxidação, portanto, não preservou a cor até o $14^{\mathrm{a}}$ dia de maturação. A embalagem a vácuo torna a carne mais escura (Realini \& Marcos, 2014). A carne embalada a vácuo apresentou valor de $\mathrm{L}^{*}$ maiores que 37 pontos a partir do sétimo dia, portanto, uma carne mais escura é considerada menos atrativa, uma vez que a cor é a característica mais importante avaliada pelo consumidor no momento da compra, podendo não apenas valorizá-la mas também depreciá-la (Eiras et al., 2017; Guerrero et al., 2018).

Tabela 3. Efeito do método de embalagem e tempo de maturação sobre a coloração da carne de bovinos terminados em confinamento

\begin{tabular}{|c|c|c|c|c|}
\hline \multirow[b]{2}{*}{ Dias } & \multicolumn{2}{|c|}{ Métodos de embalagens } & \multirow[b]{2}{*}{$\mathrm{EPM}^{*}$} & \multirow[b]{2}{*}{$\mathrm{P}>$ Valor } \\
\hline & Filme & Vácuo & & \\
\hline \multicolumn{5}{|l|}{$\overline{\mathrm{L}^{*}}$} \\
\hline 1 & $38,81 \mathrm{~A}$ & $38,90 \mathrm{~A}$ & 0,206 & 0,921 \\
\hline 3 & $41,24 \mathrm{~B}$ & $38,78 \mathrm{~A}$ & 0,233 & 0,333 \\
\hline 7 & $38,80 \mathrm{~A}$ & $39,12 B$ & 0,282 & 0,122 \\
\hline 14 & $35,80 \mathrm{C}$ & $39,76 \mathrm{C}$ & 0,258 & 0,001 \\
\hline$\overline{\mathrm{EPM}}$ & 0,213 & 0,266 & & \\
\hline $\mathrm{P}<$ Valor & 0,001 & 0,001 & & \\
\hline \multicolumn{5}{|l|}{$\mathrm{a}^{*}$} \\
\hline 1 & $12,72 \mathrm{~A}$ & $12,72 \mathrm{~A}$ & 0,193 & 0,982 \\
\hline 3 & $13,32 \mathrm{~B}$ & $12,68 \mathrm{~A}$ & 0,140 & 0,255 \\
\hline 7 & $13,85 \mathrm{~B}$ & $12,64 \mathrm{~A}$ & 0,165 & 0,277 \\
\hline 14 & $14,48 \mathrm{C}$ & $14,61 \mathrm{~B}$ & 0,193 & 0,512 \\
\hline$\overline{\mathrm{EPM}}$ & 0,180 & 0,161 & & \\
\hline $\mathrm{P}<$ Valor & 0,001 & 0,001 & & \\
\hline \multicolumn{5}{|l|}{$\overline{\mathrm{b}^{*}}$} \\
\hline 1 & 12,30 & 12,28 & 0,163 & 0,922 \\
\hline 3 & 13,88 & 11,60 & 0,132 & 0,001 \\
\hline 7 & 13,39 & 11,77 & 0,123 & 0,001 \\
\hline 14 & 12,57 & 13,65 & 0,173 & 0,001 \\
\hline$\overline{\mathrm{EPM}}$ & 0,155 & 0,142 & & \\
\hline $\mathrm{P}<$ Valor & 0,235 & 0,244 & & \\
\hline
\end{tabular}

*Erro padrão da média. Médias seguidas de letras maiúsculas nas colunas são deferentes $(\mathrm{P}<0,05)$. 
O tempo de maturação reduziu o valor de $\mathrm{L}^{*}$ para as carnes embaladas em papel filme, mas não teve o mesmo efeito para a carne embalada a vácuo. Desta forma, a embalagem a vácuo protege a deterioração da carne bovina, com valor superior a 38,7 pontos. Valor de $\mathrm{L}^{*}$ inferior a 38 caracteriza carnes mais escuras e, portanto, menos atraente ao consumidor. A redução do valor de $\mathrm{L}^{*}$ na carne quando relacionada ao período de maturação é devido à oxidação lipídica e proteica da carne.

$\mathrm{O}$ valor de $\mathrm{a}^{*}$ mede a coloração vermelha da carne. Desta forma, valor mais elevado de $\mathrm{a}^{*}$ significa uma carne mais vermelha, portanto, mais atraente ao consumidor (Faustman et al., 2010; Mancini \& Hunt, 2005). $\mathrm{O}$ valor de $\mathrm{a}^{*}$ é muito mais variável em comparação ao valor de $\mathrm{L}^{*}$. $\mathrm{O}$ valor de $\mathrm{a}^{*}$, por exemplo, pode variar de 10 a 18 (Page et al., 2001).

Os valores de $\mathrm{a}^{*}$, antes e após da maturação foram semelhantes $(\mathrm{P}>0,05)$ para as carnes dos dois métodos de embalagens. Em condições semelhantes de alimentação, manejo e grau de sangue dos animais, o valor de a* pode variar de 11 (Monteschio et al., 2017) a 18 (Rivaroli et al., 2016; Rivaroli et al., 2020), com valores intermediários entre 14 e 17 (Fugita et al., 2018; Ornaghi et al., 2020). Desta forma, os valores observados até o sétimo dia de maturação e para as carnes embaladas a vácuo, neste experimento, estão próximos aos valores observados para bovinos mestiços (Europeus vs. Zebus) em semelhantes condições de alimentação e manejo.

O tempo de maturação provocou um aumento nos valores de a* nas carnes embaladas em bandeja com papel filme e a vácuo. Neste contexto, a maturação torna a carne mais atraente ao consumidor uma vez que valor mais elevado de $\mathrm{a}^{*}$ representa uma cor mais avermelhada, portanto, mais atraente ao consumidor (Eiras et al., 2017; Guerrero et al., 2018).

O valor de $b^{*}$ caracteriza a cor mais ou menos amarelada da carne (Page et al., 2001). Valor mais elevado de $b^{*}$ da carne bovina caracteriza carne mais amareladas, menos escuras e, portanto, mais atraente ao consumidor.

Antes da maturação e 14 dias após, o método de embalagem não teve efeito no valor de b* (Tabela 3). No entanto, no terceiro e sétimo dia de maturação, o valor de $b^{*}$ foi maior $(\mathrm{P}<0,05)$ para as carnes embaladas em papel filme. Desta forma, a embalagem em papel filme protegeu a carne de uma deterioração do valor de $\mathrm{b}^{*}$, tornando a carne mais atraente ao consumidor.

\section{Textura (valores de WBSF) da carne}

O método de embalagem (bandeja com papel filme ou a vácuo) não teve efeito $(\mathrm{P}>0,05)$ na evolução da maciez da carne antes da maturação ou com 3, 7 e 14 dias de maturação (Tabela 3). Desta forma, o método de embalagem não determina uma alteração nas estruturas mio fibrilar da carne.

Por outro lado, os valores do WBSF (maciez da carne) reduziram-se $(\mathrm{P}<0,05)$ com o tempo de maturação das carnes dos animais de forma semelhante para os dois métodos de embalagens adotados. Os valores passaram de 9,23 kgf antes da maturação para 4,96 kgf após 14 dias de maturação. Desta forma, a maturação é um processo que melhora a maciez da carne. Os valores observados antes da maturação $(9,23 \mathrm{kgf})$ mostra uma carne dura para os padrões da carne bovina, este padrão pode ser atribuído à razão do stress antes e pósabate e, ainda, a raça dos animais. Neste experimento, os animais eram mestiços entre europeus e zebus. De modo geral, grau de sangue zebu caracteriza uma carne menos macia em razão do complexo calpaína e calpastatina. Ainda, com sete dias de maturação o valor de WBSF está em 6,0 kgf para a carne dos dois sistemas de embalagens. Segundo Campo et al. (2006) a carne só pode ser considerada tenra quando o valor de WBSF está abaixo de cinco. Neste caso, as carnes consideradas macias foram observadas somente com 14 dias de maturação. Todavia, a carne mais atraente ao consumidor é com 7 dias de maturação.

Tabela 3. Efeito do método de embalagem e tempo de maturação sobre a textura da carne de bovinos terminados em confinamento

\begin{tabular}{llllr}
\hline \multirow{2}{*}{ Dias } & \multicolumn{2}{c}{ Métodos de embalagens } & & \multirow{2}{*}{ EPM } \\
\cline { 2 - 3 } & Filme & Vácuo & & P Valor \\
\hline 1 & $8,89 \mathrm{~A}$ & $8,89 \mathrm{~A}$ & 0,423 & 0,982 \\
3 & $6,76 \mathrm{~B}$ & $7,36 \mathrm{~B}$ & 0,339 & 0,242 \\
7 & $5,83 \mathrm{~B}$ & $5,88 \mathrm{~B}$ & 0,245 & 0,663 \\
14 & $4,90 \mathrm{C}$ & $5,13 \mathrm{C}$ & 0,288 & 0,342 \\
\hline EPM & 0,320 & 0,223 & & \\
P Valor & 0,001 & 0,001 & & \\
\hline
\end{tabular}

\footnotetext{
*Erro padrão da média. Médias seguidas de letras maiúsculas nas colunas são deferentes $(\mathrm{P}<0,05)$.
} 


\section{Oxidação lipídica da carne}

A oxidação lipídica foi medida como a concentração de malonaldeído (MDA) por kg de carne. A oxidação foi quantificada usando a substância reativa do ácido tiobarbitúrico (valores de TBARS).

$\mathrm{O}$ método de embalagem não teve efeito $(\mathrm{P}>0,05)$ na oxidação lipídica antes e durante a maturação por 14 dias (Tabela 4). Desta forma, o método de embalagem da carne (papel filme ou a vácuo) não interfere na oxidação lipídica durante a maturação.

Tabela 4. Efeito do método de embalagem e tempo de maturação sobre a oxidação lipídica da carne de bovinos terminados em confinamento

\begin{tabular}{llllc}
\hline \multirow{2}{*}{ Dias } & \multicolumn{2}{c}{ Métodos de embalagens } & & \multirow{2}{*}{ EPM $>$ Valor } \\
\cline { 2 - 3 } & Filme & Vácuo & & 0,805 \\
3 & $0,48 \mathrm{~A}$ & $0,48 \mathrm{~A}$ & 0,027 & 0,729 \\
7 & $0,37 \mathrm{~A}$ & $0,50 \mathrm{~A}$ & 0,015 & 0,775 \\
14 & $0,50 \mathrm{~A}$ & $0,56 \mathrm{~A}$ & 0,025 & 0,891 \\
\hline EPM & $0,71 \mathrm{~B}$ & $0,65 \mathrm{~B}$ & 0,036 & \\
$\mathrm{P}<$ Valor & 0,032 & 0,022 & & \\
\hline
\end{tabular}

*Erro padrão da média. Médias seguidas de letras maiúsculas nas colunas são deferentes $(\mathrm{P}<0,05)$.

A oxidação lipídica da carne antes da maturação e até o sétimo dia foi semelhante com valores entre 0,48 a 0,53 MDA $/ \mathrm{kg}^{-1}$ de carne crua. No entanto, no décimo quarto dia os valores de MDA foram maiores $(0,68$ $\mathrm{MDA} / \mathrm{kg}^{-1}$ de carne crua) mostrando a oxidação da carne que ocorre com a maturação.

Os valores iniciais de TBARS (0,48 MDA $/ \mathrm{kg}^{-1} \mathrm{de}$ carne crua) podem ser considerados elevados. De modo geral, valores de TBARS da carne de bovinos terminados em confinamento situam-se entre 0,10 e 0,25 (Guerrero et al., 2018; Monteschio et al., 2017; Rivaroli et al., 2016; Rivaroli et al., 2020). Todavia, Ornaghi et al. (2020) também observaram valores de TBARS próximos de $0,40 \mathrm{mg}^{\text {de }} \mathrm{MDA}^{-1} / \mathrm{kg}$ de carne crua. Os valores observados para o TBARS após 14 dias de maturação foram baixos (menores do que $0,70 \mathrm{mg}$ de $\mathrm{MDA} / \mathrm{kg}^{-1}$ de carne crua).

Segundo Campo et al. (2006) valores de TBARS próximos de 2,0 pode ser considerado o limite para aceitabilidade da carne pelos consumidores No geral, a baixa oxidação lipídica observada nas carnes quando aplicada os dois métodos de embalagens e para os dias de maturação pode ser explicado pelo histórico dos animais. Os animais entraram no confinamento com aproximadamente 20 meses de idade. Antes do período de confinamento os animais foram recriados a pasto em condições tropicais. Durante este período, há um grande acúmulo de $\beta$-caroteno que protege a carne contra a oxidação de lipídeos durante curto período de confinamento, como observado em outros estudos (Guerrero et al., 2018; Monteschio et al., 2017; Rivaroli et al., 2016; Rivaroli et al., 2020). O período de confinamento deste estudo foi de 84 dias.

\section{Atividade antioxidante da carne}

Foram usadas três metodologias para a determinação da atividade antioxidante da carne bovina para as duas embalagens e para os tempos de maturação (Tabela 5). Os métodos usados para a embalagem das carnes não detectaram diferença $(\mathrm{P}>0,05)$ na atividade antioxidante (Tabela 5). Desta forma, os métodos de embalagens não interferiram na atividade antioxidante da carne bovina.

Por outro lado, com a metodologia de DPPH e FRAP, o tempo de maturação não teve efeito na atividade antioxidante na carne bovina. No entanto, usando a metodologia de ABTS, foi observada uma melhor atividade antioxidante nas carnes maturados por sete e 14 dias em comparação com as carnes maturadas com 3 dias e antes da maturação, primeiro dia.

Os valores observados neste estudo com as metodologias de DPPH (média de 23\%), ABTS (média de $40 \%$ e FRAP (média de 0,09 mg de EAG/g ${ }^{-1}$ carne) estão próximos dos valores observados em outros estudos em pescado (Vital et al., 2018) ou carne bovina (Monteschio et al., 2017). Os animais usados neste estudo foram recriados a pasto e posteriormente confinados por 84 dias. As pastagens tropicais são ricas em $\beta$ caroteno que poderiam favorecer a atividade antioxidante na carne por um longo período de tempo (Descalzo et al., 2007; Pouzo et al., 2016). 
A atividade antioxidante na carne bovina é tema bastante recente nas pesquisas (Kempinski et al., 2017; Monteschio et al., 2017; Vital et al., 2018). Algumas estratégias têm sido estudadas para impedir ou reduzir a oxidação da carne embalada e exposta em vitrine para sua comercialização. Estas estratégias podem ser a utilização de antioxidantes na alimentação animal (Guerrero et al., 2018; Prado et al., 2016; Rivaroli et al., 2017; Rivaroli et al., 2020), no momento da embalagem ou processamento da carne, como o uso de coberturas comestíveis (Vital et al., 2016) ou aspersão dos antioxidantes na carne ou sua impregnação na embalagem.

Tabela 5. Efeito do método de embalagem e tempo de maturação sobre a atividade antioxidante da carne de bovinos terminados em confinamento

\begin{tabular}{|c|c|c|c|c|}
\hline \multirow[b]{2}{*}{ Dias } & \multicolumn{2}{|c|}{ Métodos de embalagens } & \multirow[b]{2}{*}{$\mathrm{EPM}^{*}$} & \multirow[b]{2}{*}{$\mathrm{P}>$ Valor } \\
\hline & Filme & Vácuo & & \\
\hline \multicolumn{5}{|l|}{$\overline{\mathrm{DPPH}}, \%$} \\
\hline 1 & 22,73 & 22,73 & 0,966 & 0,541 \\
\hline 3 & 24,49 & 26,31 & 1,149 & 0,180 \\
\hline 7 & 23,96 & 22,85 & 0,723 & 0,761 \\
\hline 14 & 23,27 & 23,30 & 0,824 & 0,659 \\
\hline EPM & 0,998 & 0.912 & & \\
\hline $\mathrm{P}<$ Valor & 0,532 & 0,666 & & \\
\hline \multicolumn{5}{|l|}{$\overline{\mathrm{ABTS}, \%}$} \\
\hline 1 & $45,61 \mathrm{~A}$ & $45,61 \mathrm{~A}$ & 1,830 & 0,765 \\
\hline 3 & $48,45 \mathrm{~A}$ & $48,73 \mathrm{~A}$ & 1,891 & 0,651 \\
\hline 7 & $60,99 \mathrm{~B}$ & $60,72 \mathrm{~B}$ & 1,777 & 0,554 \\
\hline 14 & $63,86 \mathrm{~B}$ & $68,08 \mathrm{~B}$ & 1,645 & 0,556 \\
\hline EPM & 2,334 & 2,441 & & \\
\hline $\mathrm{P}<$ Valor & 0,001 & 0,001 & & \\
\hline \multicolumn{5}{|c|}{ FRAP, mg EAG/g ${ }^{-1}$ carne } \\
\hline 1 & 0,09 & 0,08 & 0,001 & 0,976 \\
\hline 3 & 0,09 & 0,08 & 0,001 & 0,955 \\
\hline 7 & 0,09 & 0,08 & 0,001 & 0,912 \\
\hline 14 & 0,09 & 0,08 & 0,001 & 0,913 \\
\hline$\overline{\mathrm{EPM}}$ & 0,001 & 0,001 & & \\
\hline $\mathrm{P}<$ Valor & 0,889 & 0,923 & & \\
\hline
\end{tabular}

*Erro padrão da média. Médias seguidas de letras maiúsculas nas colunas são deferentes $(\mathrm{P}<0,05)$.

\section{Conclusão}

A metodologia usada para a embalagem tem efeito no $\mathrm{pH}$ e atratividade (coloração) da carne para os consumidores. Carnes embaladas a vácuo protegem o valor do $\mathrm{pH}$ e coloração da carne, mas não tem efeito na maciez, oxidação lipídica e atividade antioxidante. Desta forma, a metodologia escolhida para embalar a carne bovina depende de outros fatores, como, o custo, conveniência, disponibilidade e aceitabilidade pelo consumidor. Por outro lado, a maturação melhorou o pH, a maciez e evitou a perda da coloração da carne de bovinos terminados em confinamento.

\section{Agradecimentos}

Este projeto foi financiado pela Fundação Araucária do estado do Paraná e o Conselho Nacional de Desenvolvimento Científico e Tecnológico $(\mathrm{CNPq})$. Os autores agradecem a Alltech do Brasil pelo financiamento de parte do projeto e o Sr. Edson Gaudêncio pela cessão dos animais em comodato (Santo Antônio da Platina, Paraná, Brasil). Os nomes das empresas ou nomes comerciais dos produtos são mencionados nessa publicação somente com o objetivo de informações técnicas e não implica em recomendação ou endosso por parte do Departamento de Zootecnia da Universidade Estadual de Maringá. Maringá, estado do Paraná, Brasil.

\section{Conflito de interesses}

Declaramos para todos os fins de direito que não existe conflito de interesses entre as partes. 


\section{Referências bibliográficas}

Campo, M. M., Nute, G. R., Hughes, S. I., Enser, M., Wood, J. D., \& Richardson, R. I. (2006). Flavour perception of oxidation in beef. Meat Science, 72(2), 303-311. https://doi.org/http://dx.doi.org/10.1016/j.meatsci.2005.07.015.

Descalzo, A. M., Rossetti, L., Grigioni, G., Irurueta, M., Sancho, A. M., Carrete, J., \& Pensel, N. A. (2007). Antioxidant status and odour profile in fresh beef from pasture or grain-fed cattle. Meat Science, 75(2), 299-307. https://doi.org/http://dx.doi.org/10.1016/j.meatsci.2006.07.015

Eiras, C. E., Guerrero, A., Valero, M. V, Pardo, J. A., Ornaghi, M. G., Rivaroli, D. C., Sañudo, C., \& Prado, I. N. (2017). Effects of cottonseed hulls levels in the diet and aging time on visual and sensory meat acceptability from young bulls finished in feedlot. Animal, 11(3), 529-537. https://doi.org/https://dx.doi.org/10.1017/S1751731116001749.

Faustman, C., Sun, Q., Mancini, R., \& Suman, S. P. (2010). Myoglobin and lipid oxidation interactions: Mechanistic bases and control. Meat Science, 86(1), 86-94. https://doi.org/http://dx.doi.org/10.1016/j.meatsci.2010.04.025.

Françozo, M. C., Do Prado, I. N., Cecato, U., Valero, M. V., Zawadzki, F., Ribeiro, O. L., Do Prado, R. M., \& Visentainer, J. V. (2013). Growth performance, carcass characteristics and meat quality of finishing bulls fed crude glycerin- supplemented diets. Brazilian Archives of Biology and Technology, 56(2). https://doi.org/10.1590/S1516-89132013000200019

Fugita, C. A., Prado, R. M., Valero, M. V., Bonafé, E. G., Carvalho, C. B., Guerrero, A., Sañundo, C., \& Prado, I. N. (2018). Effect of the inclusion of natural additives on animal performance and meat quality of crossbred bulls (Angus vs. Nellore) finished in feedlot. Animal Production Science, 58(11), 20762083. https://doi.org/https://doi.org/10.1071/AN16242.

Guerrero, A., Rivaroli, D. C., Sañudo, C., Campo, M. M., Valero, M. V., Jorge, A. M., \& Prado, I. N. (2018). Consumer acceptability of beef from two sexes supplemented with essential oil mix. Animal Production Science, 58(9). https://doi.org/10.1071/AN15306

Hocquette, J.-F. (2015). Is it possible to save the environment and satisify consumers with artificial meat? Journal of Integrative Agriculture, 2(14), 206-207.

Honikel, K. O. (1998). Reference methods for the assessment of physical characteristics of meat. Meat Science, 49(4), 447-457.

Kempinski, E. M. B. C., Vital, A. C. P., Monteschio, J. O., Alexandre, S., Nascimento, K. F., Madrona, G. S., Mikcha, J. M. G., \& Prado, I. N. (2017). Development and quality evaluation of infant food with oregano essential oil for children diagnosed with cerebral palsy. LWT - Food Science and Technology, 84. https://doi.org/10.1016/j.lwt.2017.06.016

Kerry, J. P. (2014). Chapter 23 - New Packaging Technologies, Materials and Formats for Fast-Moving Consumer Products. In J. H. Han (Ed.), Innovations in Food Packaging (pp. 549-584). Academic Press. https://doi.org/http://dx.doi.org/10.1016/B978-0-12-394601-0.00023-0

Li, W., Hydamaka, A. W., Lowry, L., \& Beta, T. (2009). Comparison of antioxidant capacity and phenolic compounds of berries, chokecherry and seabuckthorn. Central European Journal of Biology, 4(4), 499506. https://doi.org/http://dx.doi.org/10.2478/s11535-009-0041-1.

Maggioni, D., Prado, I. N., Zawadzki, F., Valero, M. V, Marques, J. A., Bridi, A. M., Moletta, J. L., \& Abrahão, J. J. S. (2012). Grupos genéticos e graus de acabamento sobre qualidade da carne de bovinos. Semina: Ciências Agrárias, 33(1), 391-402. https://doi.org/http://dx.doi.org/10.5433/16790359.2012v33n1p391.

Mancini, R. A., \& Hunt, M. C. (2005). Current research in meat color. Meat Science, 71(1), 100-121. https://doi.org/http://dx.doi.org/10.1016/j.meatsci.2005.03.003.

Monteschio, J. O., Souza, K. A., Vital, A. C. P., Guerrero, A., Valero, M. V., Kempinski, E. M. B. C., Barcelos, V. C., Nascimento, K. F., \& Prado, I. N. (2017). Clove and rosemary essential oils and encapsuled active principles (eugenol, thymol and vanillin blend) on meat quality of feedlot-finished heifers. Meat Science, 130, 50-57. https://doi.org/http://dx.doi.org/10.1016/j.meatsci.2017.04.002.

Ornaghi, M. G., Guerrero, A., Vital, A. C. P., Souza, K. A., Passetti, R. A. C., Mottin, C., Castilho, R. C., Sañudo, C., \& Prado, I. N. (2020). Improvements in the quality of meat from beef cattle fed natural additives. Meat $\quad$ Science, $163(108059), \quad$ 1-9. https://doi.org/http://dx.doi.org/10.1016/j.meatsci.2020.108059. 
Page, J. K., Wulf, D. M., \& Schwotzer, T. R. (2001). A survey of beef muscle color and pH. Journal of Animal Science, 79(3), 678-687.

Pouzo, L. B., Descalzo, A. M., Zaritzky, N. E., Rossetti, L., \& Pavan, E. (2016). Antioxidant status, lipid and color stability of aged beef from grazing steers supplemented with corn grain and increasing levels of flaxseed. Meat Science, 111, 1-8.

Prado, I. N., Cruz, O. T. B., Valero, M. V., Zawadzki, F., Eiras, C. E., Rivaroli, D. C., Prado, R. M., \& Visentainer, J. V. (2016). Effects of glycerin and essential oils (Anacardium occidentale and Ricinus communis) on the meat quality of crossbred bulls finished in a feedlot. Animal Production Science, 56(12). https://doi.org/10.1071/AN14661

Re, R., Pellegrini, N., Proteggente, A., Pannala, A., Yang, M., \& Rice-Evans, C. (1999). Antioxidant activity applying an improved ABTS radical cation decolorization assay. Free Radical Biology and Medicine, 26(9), 1231-1237.

Realini, C. E., \& Marcos, B. (2014). Active and intelligent packaging systems for a modern society. Meat Science, 98(3), 404-419. https://doi.org/http://dx.doi.org/10.1016/j.meatsci.2014.06.031.

Rivaroli, D.C., Guerrero, A., Valero, M. V., Zawadzki, F., Eiras, C. E., Campo, M. D. M., Sañudo, C., Jorge, A. M., \& Prado, I. N. (2016). Effect of essential oils on meat and fat qualities of crossbred young bulls finished in feedlots. Meat Science, 121. https://doi.org/10.1016/j.meatsci.2016.06.017

Rivaroli, D C, Ornaghi, M. G., Mottin, C., Prado, R. M., Ramos, T. R., Guerrero, A., Jorge, A. M., \& Prado, I. N. (2017). Essential oils in the diet of crossbred (1/2 Angus vs. $1 / 2$ Nellore) bulls finished in feedlot on animal performance, feed efficiency and carcass characteristics. Journal of Agricultural Science, 9(10), 205-212. https://doi.org/http://dx.doi.org/10.5539/jas.v9n10p205-212.

Rivaroli, Dayane Cristina, Campo, M. M., Sañudo, C., Guerrero, A., Jorge, A. M., Vital, A. C. P., Valero, M. V., Prado, R. M., \& Prado, I. N. (2020). Effect of an essential oils blend on meat characteristics of crossbred heifers finished on a high-grain diet in a feedlot. Animal Production Science, 60(4), 595-602. https://doi.org/https://doi.org/10.1071/AN18620.

Rotta, P. P., Valadares Filho, S. C., Engle, T. E., Costa e Silva, L. F., Sathler, D. F. T., Prado, I. N., Bonafé, E. G., Zawadzki, F., \& Visentainer, J. V. (2014). The impact of dietary sugarcane addition to finishing diets on performance, apparent digestibility, and fatty acid composition of Holstein $\times$ Zebu bulls. Journal of Animal Science, 92(6). https://doi.org/10.2527/jas.2013-7251

Vital, A. C. P., Guerrero, A., Kempinski, E. M. B. C., Monteschio, J. O., Sary, C., Ramos, T. R., Campo, M. M., \& Prado, I. N. (2018). Consumer profile and acceptability of cooked beef steks with edible and active coating containing oregano and rosemary essential oils. Meat Science, 143, 153-158. https://doi.org/https://dx.doi.org/10.1016/j.meatsci.2018.04.035.

Vital, A. C. P., Guerrero, A., Monteschio, J. D. O., Valero, M. V., Carvalho, C. B., Abreu Filho, B. A., Madrona, G. S., \& Prado, I. N. (2016). Effect of edible and active coating (with rosemary and oregano essential oils) on beef characteristics and consumer acceptability. PLoS ONE, 11(8). https://doi.org/10.1371/journal.pone.0160535

Vital, A. C. P., Guerrero, A., Ornaghi, M. G., Kempinski, E. M. B. C., Sary, C., Monteschio, J. O., MatumotoPintro, P. T., Ribeiro, R. P., \& Prado, I. N. (2018). Quality and sensory acceptability of fish (Oreochronis niloticus) with alginate-based coating containing essential oils. Journal of Food Science and Technology, 1, 1-11. https://doi.org/http://dx.doi/org/10.1007/s13197-018-3429-y.

Zhu, Q. Y., Hackman, R. M., Ensunsa, J. L., Holt, R. R., \& Keen, C. L. (2002). Antioxidative activities of oolong tea. Journal of Agricultural and Food Chemistry, 50(23), 6929-6934.

Recebido: 26 de abril, 2020.

Aprovado: 28 de maio, 2020.

Disponível online: 24 junho, 2020.

Licenciamento: Este artigo é publicado na modalidade Acesso Aberto sob a licença Creative Commons Atribuição 4.0 (CC-BY 4.0), a qual permite uso irrestrito, distribuição, reprodução em qualquer meio, desde que o autor e a fonte sejam devidamente creditados. 